\title{
Influence of basis set on the calculated properties of $\left(\mathrm{H}_{3} \mathrm{~N}-\mathrm{HCl}\right)$
}

\author{
Zdzislaw Latajka ${ }^{\text {a) }}$ and Steve Scheiner ${ }^{\text {b),c) }}$ \\ Department of Chemistry and Biochemistry, Southern Illinois University, Carbondale, Illinois 62901
}

(Received 12 November 1984; accepted 16 January 1985)

\begin{abstract}
The structure of $\left(\mathrm{H}_{3} \mathrm{~N}-\mathrm{HCl}\right)$ is investigated by ab initio calculations using a number of different basis sets ranging from minimal to split valence. The effects of including a diffuse $s p$ shell and $d$ orbitals on $\mathrm{Cl}$ are considered as well. The geometries of the complex and the isolated subunits are fully optimized. Minimal basis sets (STO-3G, STO-6G, and MINI-1) lead to an overestimate of the interaction between the subunits. Addition of $d$ functions produces only a marginal improvement. The 3-21G, 3-21 + G, MIDI-1, and LP-31G split-valence sets erroneously predict an ion pair $\left(\mathrm{H}_{3} \mathrm{NH}^{+} \ldots{ }^{-} \mathrm{Cl}\right)$ in the equilibrium structure, a conclusion which is reversed by polarization of each basis. On the other hand, both the ion pair and $\left(\mathrm{H}_{3} \mathrm{~N} \ldots \mathrm{HCl}\right)$ complexes are identified as minima in the $4-31 \mathrm{G}$ potential. When this basis set is augmented with $d$ functions, agreement with previous calculations involving large basis sets is quite good.
\end{abstract}

\section{INTRODUCTION}

During the last several years, improvements in computational hardware and quantum chemical programs have made it feasible to study molecular interactions at the $a b$ initio level. ${ }^{1-4}$ Although use of sufficiently flexible basis sets can accurately describe the various contributions to the interaction, calculations involving these large basis sets can be extremely costly. It has therefore been necessary to seek smaller basis sets which retain a reasonable level of accuracy, particularly if geometry optimizations are required or if the system under investigation is fairly large. Previous studies have indicated that minimal and split-valence type basis sets can reproduce many features of the geometries of molecular complexes calculated with extended basis sets including polarization functions. ${ }^{1,4-6}$ While the reliability of the former basis sets has been thorougly investigated for interactions involving first-row atoms, examination of their treatment of the second-row analogs is less complete.

In this paper we analyze a variety of popular basis sets with regard to the calculated properties of the $\mathrm{H}$ bond in the $\mathrm{H}_{3} \mathrm{~N}-\mathrm{HCl}$ system. This complex poses an interesting test because previous work ${ }^{7-10}$ has indicated a good deal of sensitivity to the choice of basis set of the relative stability of the neutral complex and ion pair. Minimal basis sets investigated here include the widely used STO- $n \mathrm{G}$ series ${ }^{11}$ as well as the more recently developed MINI set of Huzinaga and coworkers. ${ }^{12}$ The split-valence $4-31 \mathrm{G}$ set ${ }^{13}$ is compared to the nominally similar $3-21 \mathrm{G},{ }^{14}$ designed primarily to provide an efficient framework for geometry optimizations; also tested is Huzinaga's MIDI set. ${ }^{12}$ The effects on the results of augmenting each basis set with $d$ functions on the $\mathrm{Cl}$ atom are studied as well as addition of a diffuse $s p$ shell to the 3-21G set. As will be shown below, the apparent similarity of basis sets can mask major discrepancies in results and extreme caution must be exercised in choosing a basis set for a given problem.

\footnotetext{
a) On leave from Institute of Chemistry, University of Wroclaw, 50-383, Poland.

b) To whom reprint requests should be addressed.

${ }^{c}$ Recipient of NIH Research Career Development Award (1982-87).
}

\section{METHODS}

All calculations were carried out using the GAUSSIAN80 package of $a b$ initio codes. ${ }^{15}$ Two minimal sets ${ }^{11}$ were investigated; STO-3G and STO-6G. Also tested was MINI$1^{12}$ which is similar to STO- $n \mathrm{G}$ except that the constraint of equal $s$ and $p$ exponents is removed. The split-valence sets studied were 3-21G, ${ }^{14} 4-31 \mathrm{G},{ }^{13}$ and MIDI- $1 .{ }^{12}$ Addition of a single set of (5) $d$ functions to the $\mathrm{Cl}$ atom is denoted by the appending of a single asterisk to each basis set notation. The exponent used for the "Pople" STO- $n$ G, 3-21 G, and 4-31G basis sets is $0.75^{16}$ while 0.514 was used for Huzinaga's MINI and MIDI sets. ${ }^{17}$ The electronic structure of the $\mathrm{Cl}$ center was also improved by addition of a diffuse $s p$ shell to the 3-21G set. One choice of the exponent for this shell is 0.049 , recommended by Dunning and Hay. ${ }^{18} \mathrm{~A}$ second choice of 0.1 comes from our own calculations minimizing the basis set superposition error in molecular interactions. ${ }^{19}$ Both values of the exponent are considered below and respectively denoted as $3-21+\mathrm{G}(s p=0.049)$ and $3-$ $21+\mathrm{G}(s p=0.1)$. In addition, the local potential basis set LP-31G included in the GAUSSIAN-80 package ${ }^{15}$ was examined as well. All geometries were fully optimized (subject to $C_{3 v}$ symmetry for the complex) using the gradient procedures contained within the program.

\section{RESULTS}

\section{Subunits}

Before presenting our results for the complex, we begin with an examination of the properties computed for the isolated subunits. The geometries optimized for $\mathrm{HCl}$ and $\mathrm{NH}_{3}$ are listed in Tables I and II, respectively, along with experimental data ${ }^{20}$ for each. With regard first to $\mathrm{HCl}$, the minimal basis sets all lead to overestimates of the bond length, particularly MINI-1. Inclusion of polarization functions reduces these exaggerations somewhat. The unpolarized splitvalence basis sets are also guilty of overestimated bond lengths but to a much smaller degree. Addition of a diffuse $s p$ shell to the 3-21G basis set does not improve the bond length but in fact leads to a further elongation. Augmentation of the split-valence basis sets with $d$ functions on $\mathrm{Cl}$ results in a 
TABLE I. Calculated properties of $\mathrm{HCl}$.

\begin{tabular}{lcc}
\hline \hline Basis set & $r(\mathrm{HCl})(\AA)$ & $\mu(\mathrm{D})$ \\
\hline STO-3G & 1.313 & 1.77 \\
STO-3G* & 1.287 & 1.15 \\
STO-6G & 1.311 & 1.78 \\
STO-6G* & 1.284 & 1.15 \\
MINI-1 & 1.346 & 1.93 \\
MINI-1* & 1.311 & 1.32 \\
3-21G & 1.293 & 1.86 \\
3-21 + G(sp $=0.049)$ & 1.296 & 1.91 \\
3-21 + G(sp $=0.10)$ & 1.301 & 1.89 \\
3-21G* & 1.266 & 1.49 \\
MIDI-1 & 1.298 & 1.85 \\
MIDI-1* & 1.280 & 1.41 \\
4-31G & 1.299 & 1.87 \\
4-31G* & 1.267 & 1.49 \\
LP-31G $^{*}$ & 1.283 & $\ldots$ \\
expt $^{*}$ & 1.274 & 1.11 \\
\hline \hline
\end{tabular}

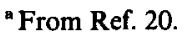

bond length in satisfactory agreement with experiment.

As noted above for $\mathrm{HCl}$, the minimal basis sets also overestimate the bond length in $\mathrm{NH}_{3}$. On the other hand, the contractions observed in the unpolarized split-valence basis sets lower the calculated bond lengths somewhat below the experimental value. The latter basis sets yield HNH bond angles about $10^{\circ}$ larger than the corresponding minimal basis set angles which are in surprisingly good accord with experiment.

Due to the well known importance of electrostatics in the SCF treatment of the $\mathrm{H}$-bonding interaction, ${ }^{1,4}$ we have included in Tables I and II the dipole moments of both molecules computed with each basis set. All the dipole moments of $\mathrm{HCl}$ obtained without polarization functions are too large, in the range 1.77-1.93 D. Again, the additional diffuse $s p$ shell in 3-21 + G has little effect upon this error. However, $d$ functions do reduce the dipole moments substantially although some exaggeration does remain with the polarized split-valence sets. In the case of $\mathrm{NH}_{3}$, most of the computed dipole moments are too large, with the exception of 4-31G. However, the agreement in the latter case is probably a fortuitous matter, due to the spuriously flat $\mathrm{NH}_{3}$ geometry (and neglect of correlation effects).

\section{Complex}

The results of geometry optimizations of the $\mathrm{H}_{3} \mathrm{~N}-\mathrm{HCl}$ complex with a variety of basis sets are presented in Table III. The first column contains the $\mathrm{H}$-bond length between

TABLE II. Calculated properties of $\mathrm{NH}_{3}$.

\begin{tabular}{lccc}
\hline \hline Basis set & $r(\mathrm{NH})(\AA)$ & $\theta(\mathrm{HNH})(\mathrm{deg})$ & $\mu(\mathrm{D})$ \\
\hline STO-3G & 1.032 & 104.2 & 1.88 \\
STO-6G & 1.028 & 104.5 & 1.89 \\
MINI-1 & 1.037 & 113.3 & 1.90 \\
3-21G & 1.003 & 112.4 & 1.75 \\
MIDI-1 & 1.001 & 112.6 & 1.86 \\
4-31G & 0.991 & 115.8 & 1.41 \\
LP-31G & 0.988 & 114.7 & $\ldots$ \\
expt & 1.011 & 106.7 & 1.48 \\
\hline \hline
\end{tabular}

a From Ref. 20. the $\mathrm{N}$ and $\mathrm{Cl}$ atoms. The bond length involving the $\mathrm{HCl}$ subunit is listed in the second column, followed by $\Delta r$, the stretch of this bond induced by formation of the complex. $r(\mathrm{~N} \cdot \mathrm{H})$ represents the distance to the H-bonding proton while $r(\mathrm{NH})$ involves the internal geometry of the $\mathrm{NH}_{3}$ subunit, as does $\theta(\mathrm{HNH})$. The interaction energy $\Delta E$ is computed as the difference in total energy between the complex and the isolated subunits. The last two columns contain the dipole moment of the complex and $\Delta \mu$, its enhancement over the sum of the moments of the isolated subunits.

From the data in the last three rows, it is clear that extended basis sets ${ }^{8-10}$ indicate a $\mathrm{H}$-bond length of about 3.3 $\AA$ and a stretch of the $\mathrm{H}-\mathrm{Cl}$ bond of some $0.02 \AA$. While these results may be expected to reasonably approximate the SCF limit, their neglect of correlation effects makes difficult a direct comparison with experiment. The present study is aimed rather at comparison of different types of basis sets within the Hartree-Fock framework. In contrast to the above data, the minimal STO- $n \mathrm{G}$ basis sets underestimate the $\mathrm{H}$-bond length by some $0.45 \AA$ and yield a $\mathrm{H}-\mathrm{Cl}$ bond elongation more than four times that computed with the extended basis sets. In addition, the energy of the $\mathrm{H}$-bond interaction is exaggerated by a factor of 2 . While the results with the MINI- 1 basis set are slightly improved over STO- $n \mathrm{G}$, similar errors remain. Inclusion of $d$ functions leads to a small improvement of all minimal basis set results, but not enough to bring the data close to the more accurate values listed at the bottom of the table.

The H-bond lengths computed with the 3-21G and 3$21+\mathrm{G}$ basis sets $(2.9 \AA)$ represent only slight improvements over the data for the minimal STO- $n \mathrm{G}$ basis sets. More importantly, the very large values of $\Delta r$ computed with these basis sets indicate a transfer of the central proton across to the $\mathrm{NH}_{3}$ subunit to form the ion pair $\left(\mathrm{H}_{3} \mathrm{NH}^{+} . .{ }^{-} \mathrm{Cl}\right)$. The MIDI- 1 basis set is guilty of the same artifact which leads also to anomolously high complexation energies $\Delta E$ and dipole moment enhancements $\Delta \mu$. On the other hand, augmenting these split-valence basis sets with $d$ functions on $\mathrm{Cl}$ reverses the above trend and the equilibrium configuration contains two neutral subunits $\left(\mathrm{H}_{3} \mathrm{~N} . . \mathrm{HCl}\right)$. Nonetheless, in comparison with the extended basis set data, the $\mathrm{H}$-bond lengths with these polarized $3-21 \mathrm{G}^{*}$ and MIDI-1* basis sets remain too short, the $\mathrm{H}-\mathrm{Cl}$ stretch too long, and the complexation energies too high.

Whereas the 3-21G and MIDI-1 split-valence basis sets lead unambiguously to the ion-pair structure, there are two minima present in the potential energy surface calculated with 4-31G. The first corresponds to an ion-pair structure much like those computed with the other split-valence sets. However, the $\mathrm{H}$-bond energy of this complex is substantially smaller than those computed with MIDI-1 and especially 3$21 \mathrm{G}$. A second very important distinction is the observation of a second 4-31G minimum which corresponds to a pair of neutral subunits. Even without polarization functions, the $\mathrm{H}$-bond length and interaction energy computed for this 4$31 \mathrm{G}$ minimum are in better accord with the extended basis sets than is $3-21 \mathrm{G}^{*}$. It may be noted that this minimum is about $0.4 \mathrm{kcal} / \mathrm{mol}$ less stable than the ion-pair structure at the Hartree-Fock level. The effects of correlation upon this 
TABLE III. Calculated properties of $\mathrm{H}_{3} \mathrm{~N}$..HCl. (All bond lengths below are in $\AA$.)

\begin{tabular}{|c|c|c|c|c|c|c|c|c|c|}
\hline Basis set & $R(\mathrm{~N} \cdot . \mathrm{Cl})$ & $r(\mathrm{HCl})$ & $\Delta r(\mathbf{H C l})$ & $r(\mathbf{N} \cdot \mathbf{H})$ & $r(\mathrm{NH})$ & $\begin{array}{l}\theta(\mathrm{HNH}) \\
\text { (deg) }\end{array}$ & $\begin{array}{c}-\Delta E \\
(\mathrm{kcal} / \mathrm{mol})\end{array}$ & $\mu(\mathrm{D})$ & $\Delta \mu(\mathrm{D})$ \\
\hline STO-3G & 2.848 & 1.411 & 0.098 & 1.437 & 1.030 & 107.0 & 18.37 & 6.92 & 3.28 \\
\hline STO-3G* & 2.890 & 1.358 & 0.071 & 1.532 & 1.030 & 106.4 & 14.90 & 5.50 & 2.48 \\
\hline STO-6G & 2.846 & 1.408 & 0.097 & 1.438 & 1.027 & 107.2 & 18.47 & 6.90 & 3.22 \\
\hline STO- $6 G^{*}$ & 2.885 & 1.356 & 0.072 & 1.528 & 1.027 & 106.7 & 14.95 & 5.51 & 2.46 \\
\hline MINI-1 & 3.021 & 1.422 & 0.076 & 1.599 & 1.043 & 111.7 & 14.35 & 6.40 & 2.57 \\
\hline MINI-1* & 3.081 & 1.361 & 0.050 & 1.720 & 1.041 & 109.6 & 11.32 & 5.02 & 1.80 \\
\hline $3-21 G$ & 2.920 & 1.790 & 0.497 & 1.130 & 1.013 & 110.7 & 25.20 & 10.77 & 6.76 \\
\hline $3-21+\mathrm{G}(s p=0.049)$ & 2.940 & 1.833 & 0.537 & 1.106 & 1.013 & 110.8 & 27.69 & 11.34 & 7.68 \\
\hline $3-21+\mathrm{G}(s p=0.10)$ & 2.917 & 1.795 & 0.494 & 1.122 & 1.013 & 110.8 & 25.72 & 10.96 & 7.32 \\
\hline $3-21 G^{*}$ & 2.991 & 1.346 & 0.080 & 1.645 & 1.007 & 111.2 & 16.66 & 5.78 & 2.14 \\
\hline MIDI-1 & 2.893 & 1.711 & 0.413 & 1.182 & 1.008 & 110.9 & 18.93 & 9.86 & 6.15 \\
\hline MIDI-1* & 3.047 & 1.344 & 0.064 & 1.703 & 1.003 & 110.0 & 13.92 & 5.46 & 2.19 \\
\hline \multirow[t]{2}{*}{ 4-31G } & 2.885 & 1.726 & 0.427 & 1.159 & 1.003 & 110.9 & 12.07 & 9.86 & 6.58 \\
\hline & 3.036 & 1.388 & 0.089 & 1.648 & 0.999 & 112.1 & 11.63 & 6.06 & 2.78 \\
\hline $4-31 G *$ & 3.184 & 1.302 & 0.035 & 1.882 & 0.997 & 112.7 & 9.31 & 4.71 & 1.81 \\
\hline LP-31G & 2.837 & 1.702 & 0.419 & 1.135 & 0.999 & 110.7 & 15.85 & & \\
\hline$[4 s 3 p 1 d / 3 s 2 p 1 d / 2 s 1 p]^{\mathrm{a}}$ & 3.281 & 1.283 & & 1.998 & & & 7.9 & & \\
\hline $6-31 \mathrm{G}^{* *}(1 p, 2 d)^{\mathrm{b}}$ & 3.297 & 1.293 & 0.023 & 2.004 & & & 9.29 & 3.90 & 1.13 \\
\hline $\mathbf{D Z}+\mathbf{P}^{\mathrm{c}}$ & 3.314 & 1.291 & 0.021 & 2.023 & & & 7.3 & 4.45 & 1.03 \\
\hline
\end{tabular}

From Ref. 8.

${ }^{b}$ From Ref. 10.

'From Ref. 9.

result were checked by second-order Møller-Plesset treatment $^{21}$ of these two geometries. Correlation was found to reverse the relative order of stability; the $\left(\mathrm{H}_{3} \mathrm{~N} \cdot . \mathrm{HCl}\right)$ complex is lower in energy than the ion pair by $1.32 \mathrm{kcal} / \mathrm{mol}$.

The ion-pair minimum disappears completely when $d$ functions are added to the 4-31G basis set. As may be seen in the appropriate row of Table III the $4-31 \mathrm{G}^{*}$ results are in quite reasonable agreement with the last three rows in all regards, including the H-bond energy. On the other hand, the local potential LP-31G set leads to the same error of ionpair formation as noted above for 3-21G and MIDI-1.

\section{CONCLUSIONS}

It has been demonstrated here that the theoretical description of the $\mathrm{H}_{3} \mathrm{~N}-\mathrm{HCl}$ complex is quite sensitive to the basis set used. The minimal sets correctly indicate that neutral subunits are involved in the interaction but the strength of the $\mathrm{H}$ bond is exaggerated. In addition, the intermolecular distance is too short and the $\mathrm{H}-\mathrm{Cl}$ bond suffers too much stretching. The errors in the results are somewhat smaller for the MINI- 1 basis than for STO- $n$ G; both types may be improved to some extent by addition of polarization functions. The calculated properties of the complex are particularly sensitive to the type of split-valence set used. 3-21G indicates a proton transfer to $\mathrm{NH}_{3}$ in the equilibrium structure consisting of an ion pair. The calculated interaction energy and dipole moment enhancement are accordingly very high. Addition of a diffuse $s p$ shell does not have any apparent effect on this tendency whereas supplementation with $d$ functions on $\mathrm{Cl}$ reverses the trend and the neutral pair becomes more stable. However, even with these polarization functions, the $\mathrm{H}$-bond energy computed with 3-21G* represents a considerable overestimate. Similar trends are noted for the MIDI-1 and MIDI-1* sets.

The situation is somewhat different with 4-31G in that both $\left(\mathrm{H}_{3} \mathrm{~N} . . \mathrm{HCl}\right)$ and $\left(\mathrm{H}_{3} \mathrm{NH}^{+} . .-\mathrm{Cl}\right)$ represent minima in the potential. The ion pair is slightly more stable at the SCF level but the order is reversed when correlation is included. The H-bond energy and geometrical features of the neutralpair complex are in fairly good agreement with results of much larger basis sets. This agreement is notably improved when 4-31G is augmented with $d$ functions on $\mathrm{Cl}$.

It therefore appears that the selection of basis set is crucial to reliable theoretical study of H-bonded systems, particularly if second-row atoms are involved. Most of the unpolarized split-valence basis sets lead to a qualitatively incorrect picture of the system considered here, belying the widely held belief that basis sets such as 3-21G provide correct structures of $\mathrm{H}$-bonded systems. ${ }^{22}$ In contrast, the interaction energy predicted by $4-31 \mathrm{G}$ is in good accord with the results of large basis sets; however, caution must be exercised in that two minima occur in the 4-31G surface. Better quantitative agreement may be obtained by supplementation with $d$ orbitals as noted previously for $\mathrm{H}_{2} \mathrm{~S}-\mathrm{HF}^{23}$

As mentioned previously, this study has been concerned largely with calculations at the Hartree-Fock level. Previous work has demonstrated that correlation effects can make major contributions to the $\mathrm{H}$-bonding interaction ${ }^{10,24}$ as well as to other phenomena. Indeed, Frisch et al. ${ }^{25}$ have demonstrated that inclusion of correlation can have a larger effect upon calculated reaction energies than various types of basis set enlargements. The reader is therefore cautioned against direct comparison of the Hartree-Fock limit with experimental data.

\section{ACKNOWLEDGMENTS}

This work was supported by grants from the National Institutes of Health (GM29391 and AM01059) and the Research Corporation.

'P. Hobza and R. Zahradnik, Weak Intermolecular Interactions in Chemistry and Biology (Elsevier, Amsterdam, 1980).

${ }^{2}$ Intermolecular Interactions, edited by $\mathrm{H}$. Ratajczak and W. J. Orville- 
Thomas (Wiley, Chichester, 1980), Vol. 1.

${ }^{3}$ P. Schuster, Angew. Chem. Int. Ed. Engl. 20, 546 (1981).

${ }^{4} \mathrm{P}$. Kollman, in Modern Theoretical Chemistry, edited by H. F. Schaefer (Plenum, New York, 1977), Vol. 4, pp. 109-152.

${ }^{s}$ W. Kolos, Theor. Chim. Acta 54, $187(1980)$.

${ }^{6} \mathrm{P}$. Hobza and R. Zahradnik, Int. J. Quantum Chem. 23, 325 (1983).

${ }^{7}$ E. Clementi, J. Chem. Phys. 46, 3851 (1967); E. Clementi and J. N. Gayles, ibid. 47, 3837 (1967).

${ }^{8}$ R. C. Raffenetti and D. H. Phillips, J. Chem. Phys. 71, 4534 (1979).

${ }^{9}$ A. Hinchliffe, J. Mol. Struct. 105, 335 (1983).

${ }^{10} \mathrm{Z}$. Latajka and S. Scheiner, J. Chem. Phys. 81, 4014 (1984).

"W. J. Hehre, R. F. Stewart, and J. A. Pople, J. Chem. Phys, 51, 2657 (1969); W. J. Hehre, R. Ditchfield, R. F. Stewart, and J. A. Pople, ibid. 52, 2769 (1970).

${ }^{12} \mathrm{H}$. Tatewaki and S. Huzinaga, J. Comput. Chem. 1, 205 (1980); Y. Sakai, H. Tatewaki, and S. Huzinaga, ibid. 2, 100 (1981).

${ }^{13}$ R. Ditchfield, W. J. Hehre, and J. A. Pople, J. Chem. Phys. 54, 724 (1971); W. J. Hehre and W. A. Lathan, ibid. 56, 5255 (1972).

${ }^{14}$ J. S. Binkley, J. A. Pople, and W. J. Hehre, J. Am. Chem. Soc. 102, 939 (1980); M. S. Gordon, J. S. Binkley, J. A. Pople, W. J. Pietro, and W. J. Hehre, ibid. 104, 2797 (1982).
${ }^{15}$ J. S. Binkley, R. A. Whiteside, R. Krishnan, R. Seeger, D. J. DeFrees, H. B. Schlegel, S. Topiol, L. R. Kahn, and J. A. Pople, GaUssian-80, Program No. 406 Quantum Chemistry Program Exchange, 1981.

${ }^{16}$ M. M. Francl, W. J. Pietro, W. J. Hehre, J. S. Binkley, M. S. Gordon, D. J. DeFrees, and J. A. Pople, J. Chem. Phys. 77, 3654 (1982).

${ }^{17}$ S. Huzinaga, J. Andzelm, M. Klobukowski, E. Radzio-Andzelm, Y. Sakai, and H. Tatewaki, Gaussian Basis Sets for Molecular Calculations (E]sevier, Amsterdam, 1984].

${ }^{18}$ T. H. Dunning, Jr. and P. J. Hay, in Modern Theoretical Chemistry, edited by H. F. Schaefer (Plenum, New York, 1977), Vol. 3, pp. 1-27.

${ }^{19} \mathrm{Z}$. Latajka and S. Scheiner, Chem. Phys. Lett. 105, 435 (1984).

${ }^{20} \mathrm{~K}$. P. Huber and G. Herzberg, Molecular Spectra and Molecular Structure (Van Nostrand Reinhold, New York, 1979), Vol. 4.

${ }^{21}$ C. Møller and M. S. Plesset, Phys. Rev. 46, 618 (1934); J. S. Binkley and J. A. Pople, Int. J. Quantum Chem. 9, 229 (1975).

${ }^{22}$ P. Hobza and R. Zahradnik, Chem. Phys. Lett. 82, 473 (1981).

${ }^{23}$ S. Scheiner, J. Chem. Phys. 78, 599 (1983).

${ }^{24}$ Z. Latajka and S. Scheiner, J. Chem. Phys. 81, 407, 2713 (1984); M. M. Szczesniak and S. Scheiner, ibid. 80, 1535 (1984); M. M. Szczesniak, S. Scheiner, and Y. Bouteiller, ibid. 81, 5024 (1984).

${ }^{25}$ M. J. Frisch, J. A. Pople, and J. S. Binkley, J. Chem. Phys. 80, 3265 (1984). 